\title{
Anti-neuronal antibodies associated with headache with neurological deficits and cerebrospinal fluid lymphocytosis
}

\author{
Murat Kürtüncü · Erdem Tüzün · Dilaver Kaya • \\ Sema Içöz • M. Barış Baslo • Mustafa Ertaş • \\ Gülş̧en Akman-Demir · Mefküre Eraksoy
}

Received: 5 July 2008/ Accepted: 24 July 2008/Published online: 12 August 2008

(C) Springer-Verlag 2008

Infectious-immunogenic mechanisms have been proposed for the pathogenesis of the syndrome of transient headache and neurological deficits with cerebrospinal fluid lymphocytosis (HaNDL) [1]. To investigate this assumption, we performed immunohistochemistry and Western blot studies with sera and cerebrospinal fluid (CSF) of two HaNDL patients. Serum and CSF IgGs of both patients showed strong reactivity with the nuclei of rat central nervous system (CNS) cells and proteins extracted from neuroblastoma cells. This reactivity was not observed with samples of a sporadic hemiplegic migraine (SHM) patient and 12 migraine patients used as controls. The CNS binding pattern of HaNDL IgG was easily distinguishable from that of other well-known anti-neuronal nuclear antibodies (ANNA) such as anti-Hu, anti-Ri, and anti-nuclear antibody (ANA). The presence of an anti-neuronal immune response may support the autoimmune hypothesis as a pathogenic factor for HaNDL and should be further investigated in larger series.

To show the presence of a possible autoimmune response in this syndrome, sera and CSF of two HaNDL patients were investigated for anti-neuronal antibodies. Serum/CSF of a SHM patient, sera from 12 migraine, 2 anti-Hu, 2 anti-Ri, 34 ANA positive patients and 30 healthy individuals were used as controls. All headache patients

M. Kürtüncü · E. Tüzün $(\bowtie) \cdot S$. İçöz $\cdot$ M. Barı̧̧ Baslo ·

M. Ertaş · G. Akman-Demir · M. Eraksoy

Department of Neurology, Istanbul University, Istanbul Faculty

of Medicine, Çapa, 34390 Istanbul, Turkey

e-mail: drerdem@yahoo.com

D. Kaya

Department of Neurology, Acrbadem University,

Istanbul, Turkey fulfilled the clinical criteria established by The International Headache Society [2].

Transient headache and neurological deficits with cerebrospinal fluid lymphocytosis patients were 35-(H1) and 38year-old (H2) men presenting with three episodes of throbbing headache, dysphasia/dysarthria, marching right hemiparesis and hemihypoesthesia (H1 only) lasting for 29 h. Their CSF revealed increased lymphocytes $\left[496 / \mathrm{mm}^{3}\right.$ (H1) and $\left.55 / \mathrm{mm}^{3}(\mathrm{H} 2)\right]$ and normal (H1) or increased (H2) protein content $(144 \mathrm{mg} / \mathrm{dl})$. A survey for viral aseptic meningoencephalitis yielded negative results for both patients. H1 had normal cranial MRI and EEG examinations and $\mathrm{H} 2$ had left fronto-parietal leptomeningeal enhancement on MRI, left temporal and parietal lobe hypoperfusion on SPECT and slowing of the left fronto-temporal activity on EEG. Both patients recovered completely with flunarizine, lamotrigine or acetylsalicylic acid. CSF samples obtained 3 months after first presentation were normal.

All patients and control sera were tested with immunohistochemistry performed with frozen rat brain sections and Western blot performed with SY5Y neuroblastoma cells separated by electrophoresis on a $4-12 \%$ polyacrylamide gel, as described previously [3]. Reactivity of the samples with the non-neuronal tissues was assessed with a kit containing primate intestine and HEp-2 cells (Euroimmun, Lübeck, Germany).

Serum and CSF IgG of both HaNDL patients reacted strongly with the nuclei of cerebral and cerebellar neurons. While anti-Hu, anti-Ri and ANA positive sera uniformly stained all cerebellar nuclei, HaNDL IgG predominantly stained the nuclei of Purkinje cells and reactivity with molecular and granular cells was significantly less intense (Fig. 1 left two columns). In contrast with anti-Hu and ANA samples, HaNDL IgG did not bind to intestinal tissue or HEp-2 cells (Fig. 1 right two columns). Three-month 
Fig. 1 The binding pattern of HaNDL patients' IgG with the rat cerebral and cerebellar nuclei is similar but somewhat distinctive from those of anti$\mathrm{Hu}$, anti-Ri and ANA positive serum samples (left two columns, original magnification $\times 100$, counterstained with hematoxylin). ANA positive sera react with the nuclei of both the widespread intestinal and HEp-2 cells, anti-Hu positive sera only react with the nuclei of intestinal neuronal cells (arrow) and anti-Ri positive and HaNDL sera do not react with any of these tissues (right two columns, original magnification $\times 100$, immunofluorescence, normal normal serum sample, $G$ granular layer, $P$ Purkinje cells, $M$ molecular layer, $A N A$ anti-nuclear antibody)

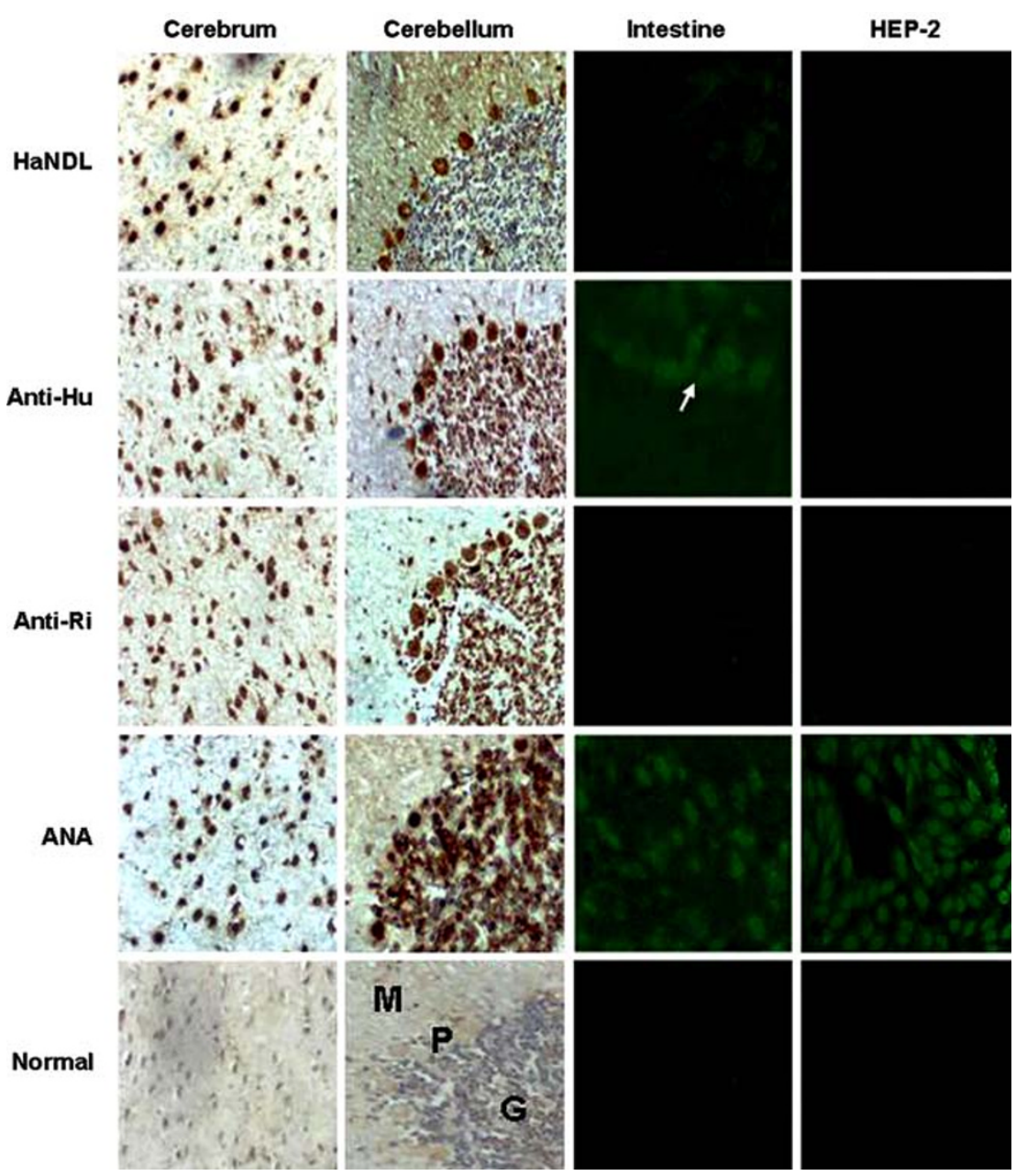

follow-up serum/CSF samples of HaNDL patients showed similar staining patterns. None of the samples from normal controls (Fig. 1 bottom row), migraine patients and the SHM patient (data not shown) exhibited immunoreactivity. Sera/CSF IgGs of both HaNDL patients but not those of the SHM or migraine patients (data not shown) yielded multiple Western blot bands (Fig. 2). However, there were no common bands for both patients.

Autoimmunity has long been proposed as a possible pathogenic factor for HaNDL [4]. The identification of novel anti-neuronal antibodies primarily directed against the nuclei of cerebral and cerebellar Purkinje cells in the sera and CSF of both HaNDL patients supports the presence of an immune response against CNS neurons and implies an abnormal humoral autoimmune reactivity in the pathogenesis of HaNDL.

Fig. 2 Western blot analysis of HaNDL patients' sera (H1, H2) showed multiple bands using the SH-SY5Y neuroblastoma cells. No common bands were retrieved. Anti-Hu and anti-Ri positive sera yielded their characteristic $35-40$ and $55-80 \mathrm{kD}$ bands, respectively $[N$ normal control, molecular size markers (in kilodaltons, $\mathrm{kD}$ ) indicated on the right]

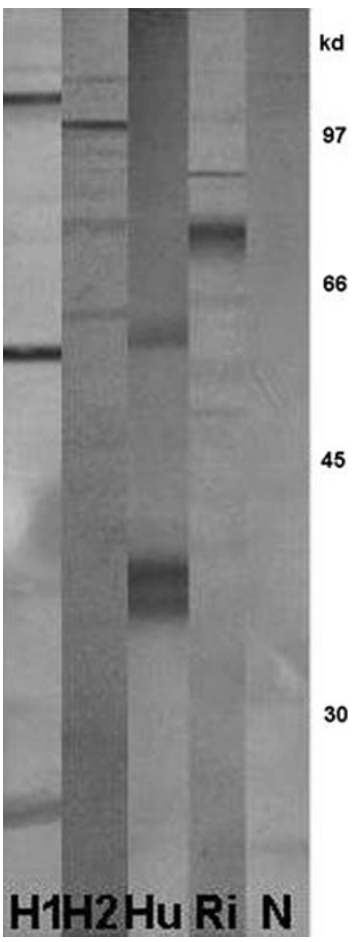




\section{Conflict of interest None.}

\section{References}

1. Gómez-Aranda F, Cañadillas F, Martí-Massó JF et al (1997) Pseudomigraine with temporary neurological symptoms and lymphocytic pleocytosis. A report of 50 cases. Brain 120:11051113
2. Headache Classification Subcommittee of the International Headache Society (2004) The international classification of headache disorders. 2nd edn. Cephalalgia 24:1-152

3. Saiz A, Arpa J, Sagasta A et al (1997) Autoantibodies to glutamic acid decarboxylase in three patients with cerebellar ataxia, lateonset insulin-dependent diabetes mellitus, and polyendocrine autoimmunity. Neurology 49:1026-1030

4. Martin-Balbuena S, Arpa-Gutierrez FJ (2007) Pseudomigraine with cerebrospinal fluid pleocytosis or syndrome of headache, temporary neurological deficit and cerebrospinal fluid. A historical review. Rev Neurol 45:624-630 